\title{
Inyección intracitoplasmática de espermatozoides, treinta años después de su implementación
}

\author{
Intracytoplasmic sperm injection, thirty years after its implementation
}

Luisa F. Calderón-Mendoza Biol', Walter D. Cardona-Maya PhD²

\begin{abstract}
Resumen: la infertilidad es una enfermedad que se caracteriza por la imposibilidad de lograr un embarazo después de más de 12 meses de relaciones sexuales; como consecuencia, existen tratamientos para manejar este trastorno. Uno de los tratamientos de reproducción asistida es la inyección intracitoplasmática de espermatozoides (ICSI), la cual fue implementada en 1992 para tratar parejas con el factor masculino asociado a la causa de infertilidad. Actualmente, esta técnica es indicada para la infertilidad sin causa aparente, fallas anteriores en los procesos de fertilización in vitro, edad materna avanzada, oocitos de mala calidad, entre otros. La inyección intracitoplasmática de espermatozoides comienza con una estimulación ovárica controlada mediante gonadotropinas y la aspiración folicular para obtener los oocitos. Simultáneamente se procesa la muestra de semen y posteriormente se realiza la microinyección del espermatozoide elegido al interior del oocito. Por otro lado, los parámetros seminales y funcionales han adquirido gran importancia debido al papel determinante en el éxito de la inyección intracitoplasmática de espermatozoides, especialmente la integridad del ADN espermático. Finalmente, la inyección intracitoplasmática de espermatozoides permite que los pacientes con alteraciones seminales tengan la posibilidad de concebir un hijo biológico. En esta revisión se describen los fundamentos de esta técnica y su relación con los parámetros seminales y la fertilidad.
\end{abstract}

Palabras clave: inyecciones de esperma intracitoplasmáticas, fertilidad, análisis de semen, técnicas reproductivas.

Abstract: Infertility is a disease characterized by the inability to achieve pregnancy after more than 12 months of sexual intercourse; as a result, there are treatments to manage this disorder. One of the treatments of assisted reproduction is the intracytoplasmic sperm injection (ICSI), which was implemented in 1992 to treat couples with male factor associated with the cause of infertility.

\footnotetext{
' Bióloga, estudiante de Maestría, Universidad CES, Centro de Medicina Reproductiva CONCEVIDAS. Medellín, Colombia ${ }^{2}$ Bacteriólogo y Laboratorista Clínico, MSc en Ciencias Básicas Biomédicas, PhD en Biología. Docente Grupo Reproducción, Departamento de Microbiología y Parasitología, Facultad de Medicina, Sede de Investigación Universitaria, Laboratorio 534, Universidad de Antioquia. Medellín, Colombia. Correo electrónico: wdario.cardona@udea.edu.co/wdcmaya@gmail.com

Conflicto de intereses: los autores declaran que no tienen conflicto de intereses

Medicina \& Laboratorio 2015; $21: 431-444$

Módulo 27 (Salud Sexual y Reproductiva), número 5. Editora Médica Colombiana S.A. $2015^{\circ}$

Recibido el 03 de septiembre de 2015; aceptado el 15 de octubre de 2015
}

Medicina \& Laboratorio Volumen 21, Números 9-10, 2015. 
Currently, this technique is indicated to unexplained infertility, previous in vitro fertilization failures, advanced maternal age, poor quality oocytes, among others. Intracytoplasmic sperm injection begins with a controlled ovarian stimulation and follicular aspiration to obtain oocytes. Simultaneously the semen sample is prepared and the microinjection of the selected spermatozoa into the oocyte is performed. On the other hand, the seminal and functional parameters have become very important due to the determinant role in the success of the intracytoplasmic sperm injection, especially sperm DNA integrity. Finally, intracytoplasmic sperm injection allows patients with seminal alterations have the possibility to conceive a biological child. In this review, the basics of this technique and its relationship with sperm parameters and fertility are described.

Keywords: Intracytoplasmic sperm injections, fertility, semen analysis, reproductive techniques.

Calderón-Mendoza LF, Cardona-Maya WD. Inyección intracitoplasmática de espermatozoides, treinta años después de su implementación. Medicina \& Laboratorio 201 5; 21: 431 -444.

$$
\mathrm{L}^{2}
$$
a infertilidad es una enfermedad que se caracteriza por la imposibilidad de lograr un embarazo después de más de 12 meses de relaciones sexuales sin protección en los días fértiles [I], que acarrea consecuencias emocionales y psicosociales para las parejas [2]. Una vez transcurrido este tiempo sin concebir es necesario consultar a un especialista y optar por el tratamiento más adecuado para tener un hijo vivo sano en casa. Existen diferentes tipos de tratamientos de reproducción asistida que se aplican a las parejas clasificados según su complejidad (baja y alta complejidad) cada una con un porcentaje de éxito diferente. En la categoría de baja complejidad se encuentran las relaciones sexuales dirigidas o coito programado y la inseminación intrauterina, y en la de alta complejidad la fertilización in vitro y la inyección intracitoplasmática de espermatozoides (véase tabla I).

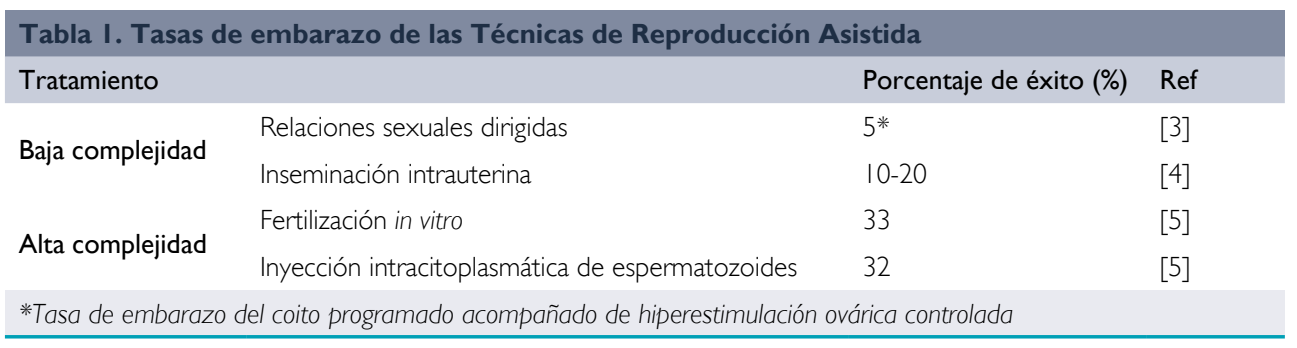

Las relaciones sexuales dirigidas o coito programado constituyen el primer paso para el manejo de la infertilidad y se recomienda a parejas que presentan infertilidad de origen desconocido y a mujeres jóvenes. El momento de las relaciones sexuales es determinado por el pico endógeno de la hormona luteinizante (LH), debido a que la ovulación se produce 24 horas después de la detección de este incremento. Sin embargo, lo más común es realizar un seguimiento ecográfico del desarrollo folicular; además, se puede realizar una estimulación ovárica específicamente en mujeres con anovulación [6].

La inseminación intrauterina es el tratamiento de primera elección para parejas infértiles, que consiste en depositar espermatozoides móviles en el útero mediante un catéter durante el período ovulatorio con el objeto de lograr un embarazo. Está técnica está indicada en mujeres con al menos una trompa de Falopio permeable y cuyas parejas tienen una muestra de semen 
con un recuento espermático mayor a tres millones de espermatozoides móviles progresivos recuperados [4,5]. Generalmente, este proceso se realiza acompañado de una estimulación ovárica controlada con dosis de gonadotropinas bajas (75 UI a I I2,5 UI) para obtener el desarrollo de varios folículos y así aumentar la probabilidad de embarazo [3,5].

Por otro lado, la fertilización in vitro fue diseñada inicialmente como tratamiento para la infertilidad asociada a la patología tubárica bilateral. No obstante, en la actualidad, tiene una amplia variedad de indicaciones como la infertilidad por factor masculino no grave, los fallos de la inseminación intrauterina (se recomienda realizar hasta cuatro intentos [4]), la disfunción ovárica y la endometriosis, entre otros [7,8].

Finalmente, la inyección intracitoplasmática de espermatozoides es uno de los tratamientos reproductivos más utilizados en la actualidad, implementada en 1992 [9] con el fin de mejorar la fecundación de los oocitos en parejas con factor masculino alterado o sin resultados satisfactorios en los ciclos de fertilización in vitro [10]. La inyección intracitoplasmática de espermatozoides consiste en inyectar un espermatozoide directamente en el citoplasma del oocito, sobrepasando la zona pelúcida y la membrana plasmática [ I l ]. Este procedimiento está indicado en parejas con una marcada alteración en los parámetros seminales como: oligozoospermia $(<15 \times 106 / \mathrm{mL})$, astenozoospermia ( $<32 \%$ de espermatozoides móviles) y teratozoospermia (<4\% de espermatozoides normales) [ I I, I2]. Teniendo en cuenta lo anterior, el objetivo de esta revisión de la literatura es describir los fundamentos de la técnica inyección intracitoplasmática de espermatozoides y su relación con los parámetros seminales y la fertilidad.

\section{Inyección intracitoplasmática de espermatozoides: ¿cuándo y por qué usarla?}

De acuerdo a la información recientemente publicada por la Sociedad Europea de Reproducción Humana y Embriología (ESHRE; del inglés, European Society of Human Reproduction and Embryology), en el año 201 I se realizaron 588.629 tratamientos de reproducción asistida en 33 países europeos, I 5 I.923 en Estados Unidos y 66.347 en Australia y Nueva Zelanda. Se estima que en este mismo año se realizaron 1.500 .000 ciclos de reproducción asistida en todo el mundo, con más de 350.000 bebés nacidos. Así mismo, la ESHRE reportó que la técnica más utilizada para la fecundación de los oocitos es la inyección intracitoplasmática de espermatozoides, lo que representa alrededor de dos tercios de los tratamientos de reproducción asistida en el mundo por encima de la fertilización in vitro convencional que representa el tercio restante. Estas proporciones varían entre los países aunque las tasas de embarazo de cada técnica son similares [I3] (véase tabla I).

De igual manera, el Comité Internacional de Monitoreo de Tecnologías de Reproducción Asistida (ICMART; del inglés, International Committee Monitoring Assisted Reproductive Technologies), en 2012, informó que el número de niños nacidos como resultado de los tratamientos de reproducción asistida ha alcanzado un estimado de cinco millones desde el nacimiento de Louise Brown en julio de 1978 [ I 4]. El cálculo se basó en el número de ciclos de fertilización in vitro e inyección intracitoplasmática de espermatozoides registrados en todo el mundo hasta el año 2008 con estimaciones adicionales para los siguientes tres años [15].

La inyección intracitoplasmática de espermatozoides se ha convertido en una opción para los pacientes sin espermatozoides en el eyaculado (azoospermia), pues si la azoospermia es de 
origen obstructiva es posible recuperar los espermatozoides por medios quirúrgicos, como la aspiración del epidídimo y la biopsia del tejido testicular. Incluso, en hombres con azoospermia no obstructiva es posible recuperar espermatozoides del eyaculado hasta en un 35\% de los casos [16], después de una búsqueda minuciosa en el precipitado obtenido al centrifugar la muestra de semen (1.600 rpm/10 min) o después de una biopsia testicular. Generalmente, la calidad morfológica de los espermatozoides recuperados en estos pacientes es baja; sin embargo, el efecto de la inyección intracitoplasmática de espermatozoides es tan alto que se han logrado obtener tasas de fecundación y de embarazo aceptables a pesar de estos inconvenientes $[17,18]$. Además, se ha observado que el éxito de la fecundación durante la inyección intracitoplasmática de espermatozoides en individuos con oligoastenoteratozoospermia es 3,9 veces mayor que si se usara la fertilización in vitro [19].

Actualmente, el uso de la inyección intracitoplasmática de espermatozoides en pacientes con parámetros seminales en el límite inferior de referencia, o incluso normales respecto a los reportados en 2010 por la Organización Mundial de la Salud [12], es común en los laboratorios de reproducción asistida [20] e incluye otras indicaciones para evitar una baja fecundación de los oocitos como: infertilidad inexplicada, oocitos de mala calidad, bajo número de oocitos, edad materna avanzada, fallos en las fertilizaciones in vitro y fecundación de oocitos criopreservados o vitrificados [2 I]. La razón fundamental para todas estas indicaciones es evitar el fracaso de la fecundación [10]; sin embargo, no existe evidencia científica que justifique el uso rutinario de la inyección intracitoplasmática de espermatozoides sin indicaciones bien establecidas [20,2 I].

Así mismo, la técnica de inyección intracitoplasmática de espermatozoides y en general los tratamientos de reproducción asistida han sido objeto de estudio para conocer su seguridad. Se conoce que la tasa de malformaciones congénitas de niños concebidos después de la inyección intracitoplasmática de espermatozoides en comparación con aquellos concebidos naturalmente no es estadísticamente significativa. Entretanto, los riesgos asociados a la inyección intracitoplasmática de espermatozoides más relevantes son los embarazos múltiples y el bajo peso al nacer; sin embargo, el subsecuente desarrollo y crecimiento de estos niños es normal [22].

\section{Inyección intracitoplasmática de espermatozoides: procedimiento complejo}

Uno de los factores determinantes del éxito de la inyección intracitoplasmática de espermatozoides es el uso de los instrumentos correctos, entre los que se encuentran un microscopio invertido con contraste de fase equipado con un sistema de contraste de modulación Hoffman y una platina calentadora para mantener los gametos a la temperatura corporal, mientras se encuentran fuera de la incubadora. Además, el microscopio debe tener acoplado el equipo de micromanipulación que consiste en soportes para las pipetas a cada lado, unidos por un tubo de teflón a los microinyectores, los cuales proporcionan fuerzas de succión y expulsión controladas. Las pipetas son de vidrio y tienen características específicas: una tiene extremos romos y se utiliza para sujetar el oocito mientras que la otra es afilada para permitir la inyección del espermatozoide. De otro lado, se encuentran los micromanipuladores para los movimientos bruscos de las pipetas y para los movimientos finos teniendo en cuenta que se pueden mover rápido o lento en varias direcciones: arriba, abajo, adelante, atrás, a la izquierda, a la derecha y 
de manera circular a lo largo del eje horizontal [17]. Además, el éxito de este procedimiento depende de la experiencia y la habilidad del embriólogo que lo realiza, teniendo en cuenta que implica una curva de aprendizaje importante [23].

\section{Procedimiento de la inyección intracitoplasmática de espermatozoides}

\section{Oocitos}

Desde hace más de 25 años se realizan ciclos con estimulación ovárica controlada utilizando gonadotropinas para optimizar los tratamientos de reproducción asistida y aumentar la tasa de embarazo [24]. Una vez se logra el desarrollo de múltiples folículos (observados por ecografía) se realiza la aspiración transvaginal de los oocitos (bajo anestesia general) 36 horas después de la aplicación de la hormona gonadotropina coriónica humana (HCG). Posteriormente, los oocitos son incubados en medio de cultivo especializado para fertilización in vitro por aproximadamente tres horas antes de remover las células del cumulus. Luego, se evalúa la madurez de los oocitos en el microscopio (200 X) [25] y se seleccionan aquellos que han alcanzado la metafase de la segunda división meiótica, es decir, el estadio de desarrollo en el cual el oocito muestra el primer cuerpo polar en el espacio perivitelino. Finalmente, los oocitos son llevados a la incubadora alrededor de una hora hasta la inyección [17].

De otro lado, es necesario realizar la preparación de la muestra de semen para realizar una mejor selección de espermatozoides con buena morfología y, de esta manera, maximizar las probabilidades de fecundación, obteniendo tantos oocitos fecundados como sea posible así como embriones para transferir eventualmente [26].

\section{Espermatozoides}

El procesamiento para la recuperación de los espermatozoides de las muestras seminales se lleva a cabo utilizando diferentes métodos dependiendo de la calidad y la movilidad de los espermatozoides observados, y consiste en seleccionar los espermatozoides con mejor movilidad y separarlos del plasma seminal, los espermatozoides inmóviles, las células inmaduras y los detritus. Este proceso da lugar a una modificación en la movilidad flagelar y al movimiento de la cabeza del espermatozoide, lo que favorece la penetración de los oocitos. Así mismo, los espermatozoides deben ser capaces de llevar a cabo la reacción acrosómica, que consiste en la liberación de enzimas que permiten la penetración a través de la zona pelúcida [27]. Por tanto, técnicas tales como los gradientes de densidad y el Swim-up, están relacionadas con la adquisición de esta capacidad de fecundar [28].

Los gradientes de densidad se fundamentan en la selección de espermatozoides para vencer la dificultad que presentan los gradientes de $90 \%$ y $45 \%$, y lograr llegar hasta el fondo del tubo después de la centrifugación; además, actúa como filtro para el plasma seminal, las células redondas, los detritus y los espermatozoides con movilidad no progresiva [27]. Para realizar este procedimiento se toma una alícuota de la muestra y se deposita suavemente sobre las capas de los gradientes discontinuos de 1,5 mL de $90 \%$ (lower) y I,5 mL de 45\% (upper), se centrifuga a $1.300 \mathrm{rpm}$ durante 20 minutos y, posteriormente, el sedimento se resuspende en medio de cultivo y se centrifuga nuevamente durante 10 minutos a 1.300 rpm. Finalmente, se 
realiza un lavado con $2 \mathrm{~mL}$ de medio, se centrifuga por 5 minutos a $1.600 \mathrm{rpm}$, y el sedimento se resuspende en un volumen de 0,3 a 0,5 mL [17] (véase figura I). Esta técnica permite una mejor recuperación de los espermatozoides móviles en las muestras oligozoospérmicas, astenozoospérmicas y con abundantes células y detritus [27].

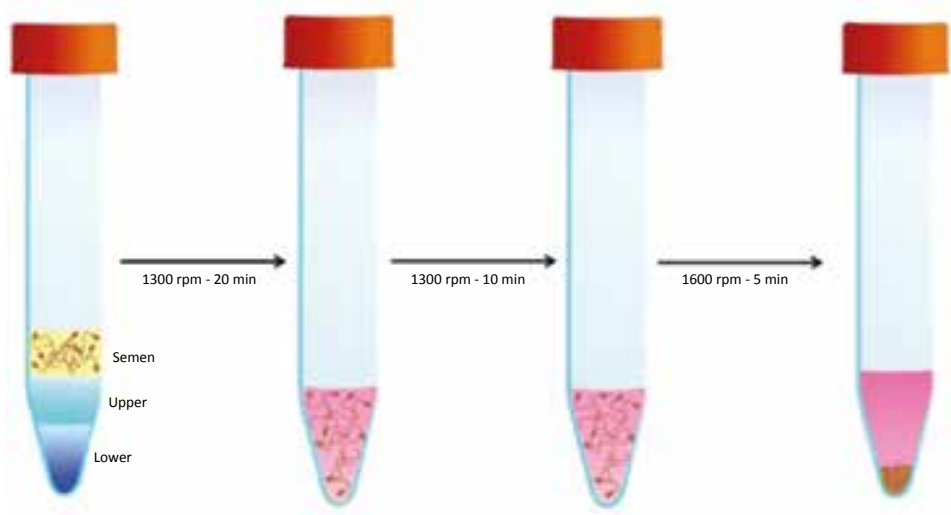

Figura I. Procedimiento de recuperación espermática usando gradientes.

De otro lado, la técnica de Swim-up se basa en el principio de que sólo los espermatozoides con buena movilidad podrán ascender al sobrenadante; es una técnica sencilla que permite una recuperación favorable en muestras normozoospérmicas [27]. Este procedimiento se lleva a cabo mediante la adición del medio de cultivo en una relación l:I con la muestra de semen, se centrifuga a 1.600 rpm por 5 minutos, se descarta el sobrenadante y se añade medio de cultivo al sedimento lentamente por la pared del tubo. Posteriormente, el tubo se incuba en un ángulo de $45^{\circ}$ de inclinación durante 45 a 60 minutos a $37^{\circ} \mathrm{C}$. Al final de este período, el tubo se retira cuidadosamente de la incubadora y el fluido que está por encima del sedimento es recuperado, el cual, por lo general, contiene espermatozoides móviles que han nadado hacia arriba. Posteriormente, se vuelve a centrifugar para concentrar los espermatozoides y se resuspende el botón en 0,5 mL de medio (véase figura 2) [17,27].

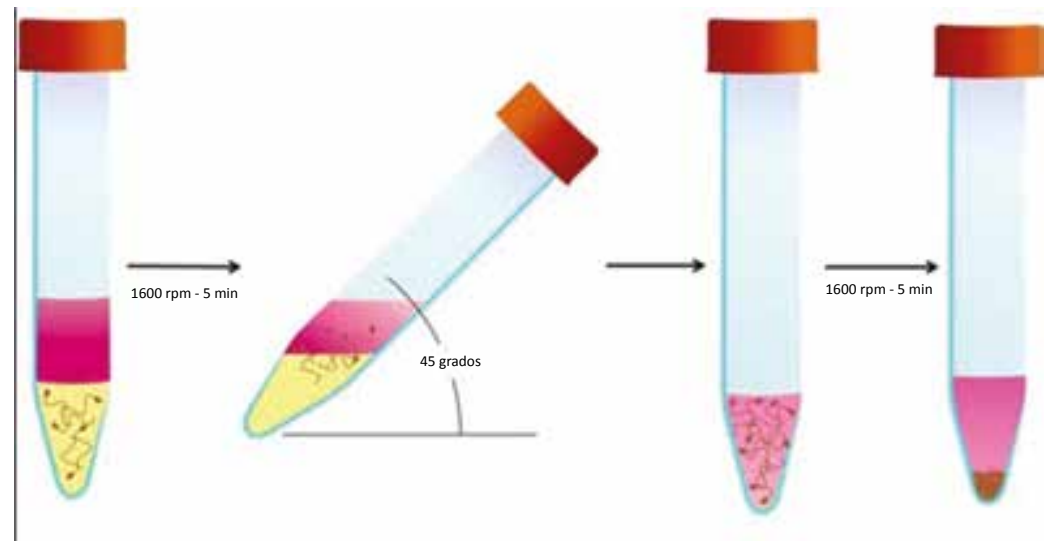

Figura 2. Procedimiento de recuperación espermática usando el método de Swim-up. 


\section{Interacción entre el espermatozoide móvil y el oocito}

La cápsula en la cual se llevará a cabo la inyección de los oocitos se prepara con gotas de $5 \mu \mathrm{L}$ del medio de cultivo con Hepes y se cubren con $5 \mathrm{~mL}$ de aceite o parafina, y se preparan las microgotas necesarias para colocar los oocitos a inyectar.

Algunas de estas microgotas se vacían y se reemplazan por polivinilpirrolidona que facilita la manipulación de los espermatozoides. Una de las microgotas con polivinilpirrolidona se utiliza sólo para purgar la pipeta de inyección antes de comenzar la búsqueda de los espermatozoides, a otra se agregan $3 \mu \mathrm{L}$ de la suspensión espermática (espermatozoides ya capacitados) y en otra más se colocan los espermatozoides elegidos para la inyección [29]. Cuando se trata de muestras seminales de mala calidad (oligozoospérmicas severas o espermatozoides provenientes de biopsia testicular) no es posible realizar las técnicas anteriormente nombradas, por lo tanto, en estos casos se debe hacer un lavado simple de la muestra que consiste en centrifugar durante 5 minutos a $1.600 \mathrm{rpm}$, descartar el fluido sobrenadante y resuspender el sedimento en $0,3 \mathrm{~mL}$ a $0,5 \mathrm{~mL}$ y, posteriormente, incubar por una hora aproximadamente a $37^{\circ} \mathrm{C}[30]$. Pasado este tiempo, se trata la muestra tal como se explicó anteriormente.

Por otro lado, la selección de los espermatozoides se realiza en el microscopio en objetivo de 400X, observando su forma, refracción de la luz y patrón de movimiento en el medio viscoso, siendo aconsejable escoger los espermatozoides que «nadan» en el borde de la gota [ I I,3 I]. Además, se prepara una microgota con sólo medio de cultivo y espermatozoides ya procesados (servirá en los casos en que los espermatozoides incubados en polivinilpirrolidona pierden totalmente su movilidad o en casos de poca concentración) [29].

Una vez elegido el espermatozoide se debe inmovilizar para facilitar su manipulación, para que no destruya las estructuras del oocito una vez esté en el interior y para facilitar la descondensación del núcleo [29]. Para realizar la inmovilización se requiere colocar la punta de la pipeta sobre el tercio proximal a la zona media del espermatozoide y, con un movimiento en sentido perpendicular al eje del espermatozoide, se dobla el flagelo hasta que éste quede angulado, se aspira el espermatozoide por la cola y se procede a la inyección [3। ]. Posteriormente, se ubica el oocito de tal manera que el cuerpo polar quede en la posición horaria de las 12 o las 6 y se sostiene el espermatozoide, luego se presiona suave y gradualmente con la pipeta de inyección sobre la zona pelúcida y, una vez traspasada la zona pelúcida, se continúa presionando la membrana del oocito y se procura que se perfile perfectamente un cono alrededor de la pipeta, moviéndola de arriba abajo hasta que se consiga, y se intenta romper la membrana. Conseguida la ruptura de la membrana se completa la microinyección, se aspira el citoplasma para cerciorarse de que la membrana está rota, se pone en contacto con el espermatozoide y este se deposita suavemente, procurando no introducir la polivinilpirrolidona en el interior del oocito [29].

\section{Inyección intracitoplasmática de espermatozoides y parámetros seminales}

\section{Parámetros seminales convencionales}

El espermograma es un análisis de la muestra de semen que se realiza de manera rutinaria en los laboratorios de andrología y es considerado como una prueba básica importante para investigar la infertilidad masculina [32], que se debe ejecutar de manera estandarizada con el fin 
de evaluar los parámetros descriptivos de un eyaculado [12]. Aunque este análisis proporciona información útil en la evaluación inicial de los pacientes que consultan por infertilidad, no revela las características funcionales de los espermatozoides que determinan el proceso de maduración requerido para la fecundación [33], no está relacionado con la capacidad fértil del hombre [34-36]; además, no discrimina entre pacientes fértiles y subfértiles [37,38]. Por lo tanto, en la actualidad algunas investigaciones sugieren la necesidad de estudiar los parámetros funcionales para establecer la calidad de la muestra de semen, específicamente en los tratamientos de reproducción asistida [39].

De acuerdo al manual para la evaluación y procesamiento del semen humano de la Organización Mundial de la Salud publicado en 2010 [12], la evaluación del semen consiste en una valoración macroscópica inicial de la licuefacción, la viscosidad, la apariencia del eyaculado, el volumen y el pH. Posteriormente, se realiza la valoración microscópica que incluye los parámetros de movilidad, viabilidad, concentración y morfología espermática. Cada uno de los parámetros seminales tiene un valor límite de referencia (percentil 5), con el cual se determina si los parámetros de una muestra de semen son normales (véase tabla 2).

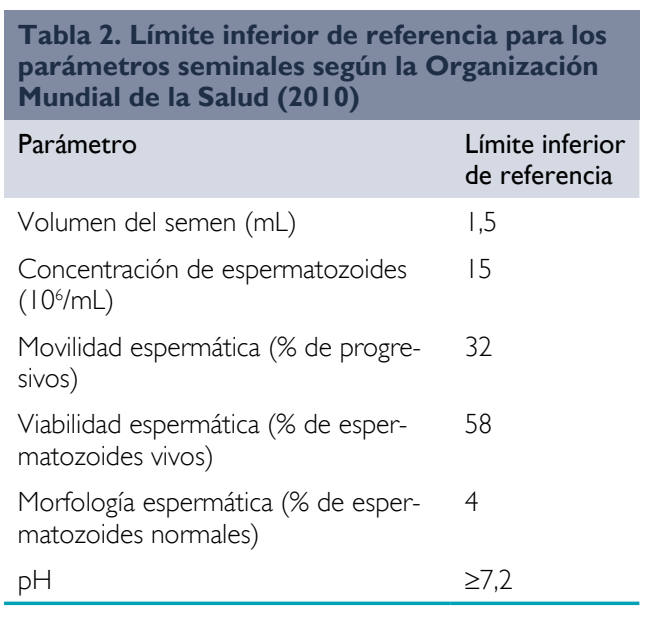

Teniendo en cuenta estos parámetros, la inyección intracitoplasmática de espermatozoides se realiza cuando el factor masculino es severo; además, en pacientes con azoospermia obstructiva y todos los tipos de azoospermia no obstructiva que requieren biopsias testiculares [40]. En un estudio realizado por Bukulmez y colaboradores (200I) [4I] se evidencia que no existen diferencias significativas en la tasa de implantación y el porcentaje de embarazo clínico cuando se inyecta con espermatozoides provenientes del eyaculado o de biopsias.

La morfología es uno de los parámetros más importantes al realizar la inyección intracitoplasmática de espermatozoides, ya que el embriólogo elige un espermatozoide para inyectar, evaluando su movilidad y su apariencia morfológica normal o adecuada. Algunos estudios indican que los resultados obtenidos por esta técnica no están relacionados con la morfología estricta, basándose en las tasas de fertilización [42] y en el desarrollo y la morfología de los blastocistos [43]. Sin embargo, De Vos y colaboradores (2003) [44] encontraron que cuando solo se dispone de espermatozoides morfológicamente anormales para inyectar, la tasa de implantación disminuye comparada con los espermatozoides normales.

En otra investigación, Keegan y colaboradores (2007) [45] evaluaron el efecto de la teratozoospermia aislada en oocitos fecundados por fertilización in vitro convencional y por inyección intracitoplasmática de espermatozoides, sin encontrar diferencias significativas en parejas con morfología anormal (<5\% de espermatozoides normales) y parejas con espermogramas normales; además, no observaron ninguna mejoría al tratar la teratozoospermia con inyección intracitoplasmática de espermatozoides. En lo que se refiere a los parámetros seminales macroscópicos como la licuefacción y la viscosidad no existe evidencia que sugiera que las muestras 
anormales afecten la tasa de fecundación, específicamente después de realizar la inyección intracitoplasmática de espermatozoides [46].

Respecto a los pacientes normozoospérmicos, de acuerdo al estudio realizado por Bhattacharya y colaboradores (200 I) [47], la inyección intracitoplasmática de espermatozoides no ofrece mejores resultados comparada con la fertilización in vitro convencional, debido a que con esta última lograron tasas de implantación y de embarazo más altas; por tal razón, los autores sugieren que en la práctica la inyección intracitoplasmática de espermatozoides se debe utilizar sólo en casos de alteración del factor masculino. Así mismo, Briton-Jones y colaboradores (2009) [48] no encontraron diferencias significativas entre la fertilización in vitro y la inyección intracitoplasmática de espermatozoides en cuanto a tasa de fecundación, tasa de desarrollo embrionario, calidad embrionaria y tasas de aneuploidias en pacientes normozoospérmicos.

Por otro lado, es necesario tener en cuenta el papel del factor femenino en los tratamientos de reproducción asistida como la inyección intracitoplasmática de espermatozoides [4I]. Es así como De Brucker y colaboradores (2014) [49] afirman que al utilizar la inyección intracitoplasmática de espermatozoides con muestras de donantes (normozoospérmicos) el éxito de la técnica, traducido como tasa de nacimiento, depende de la edad de la mujer, teniendo una tasa mayor en las pacientes entre los 20 y los 29 años.

\section{Integridad del ADN espermático}

La evaluación de la integridad del ADN de los espermatozoides ha tomado gran importancia en los últimos años, debido a su carácter predictivo en la fecundación de oocitos [50] y en el desarrollo embrionario [5 I] en los tratamientos de reproducción asistida. La etiología del daño en el ADN de los espermatozoides, caracterizado por rupturas de cadena simple y doble, es multifactorial y puede estar relacionada con factores intrínsecos, que incluyen la deficiencia de protaminas, los niveles altos de especies reactivas de oxígeno y la apoptosis abortiva, y factores extrínsecos, entre los que se encuentran la exposición ambiental, la quimioterapia y, posiblemente, algunos factores del estilo de vida [52].

La integridad del ADN de los espermatozoides es considerado como un indicador significativo del potencial fértil debido a que se ha encontrado que los hombres con altos niveles de fragmentación tienen menos probabilidades de concebir naturalmente o a través de procedimientos tales como la inseminación intrauterina y la fertilización in vitro [53], y a que no se encuentra correlacionado con los parámetros seminales evaluados en el espermograma [39,54].

Varios estudios confirman la importancia de la integridad del ADN espermático para la transmisión adecuada de la información genética [55], cuya evidencia demuestra que los altos niveles de fragmentación en el ADN no sólo retrasan el desarrollo embrionario sino que también afectan las tasas de embarazo después de realizar la inyección intracitoplasmática de espermatozoides [5।,56]; además, se ha relacionado con la pérdida recurrente del embarazo y los abortos espontáneos [57-59].

\section{Especies reactivas de oxígeno}

Las especies reactivas de oxígeno son moléculas altamente reactivas que actúan sobre los lípidos, los aminoácidos y los carbohidratos [55], y son producidas por los espermatozoides en 
pequeñas cantidades, desempeñando un papel fisiológico que promueve la función espermática para la fertilización; sin embargo, el exceso de la producción de especies reactivas de oxígeno por los espermatozoides anormales o los leucocitos seminales pueden conducir a un daño oxidativo [60]. La sobreproducción de especies reactivas de oxígeno trae como consecuencia la disminución de la movilidad espermática y el daño en el ADN, lo cual se considera como una causa importante de la infertilidad. Así mismo, afecta negativamente la tasa de fecundación en los ciclos de fertilización in vitro y de la inyección intracitoplasmática de espermatozoides [6।]. Por otro lado, este parámetro funcional ha tomado gran importancia clínica debido a su carácter tanto diagnóstico como pronóstico, específicamente en los casos de infertilidad idiopática masculina [54,62].

\section{Logros de la implementación de la inyección intracitoplasmática de espermatozoides}

La implementación de la inyección intracitoplasmática de espermatozoides en los laboratorios de reproducción asistida ha permitido que pacientes con muestras seminales anormales logren concebir un embarazo, existiendo actualmente varios estudios que lo corroboran. Incluso, en pacientes con azoospermia obstructiva y no obstructiva se han alcanzado tasas de embarazo favorables utilizando los espermatozoides provenientes de biopsias testiculares criopreservadas [18,63,64]. Así mismo, Goswani y colaboradores (2015) [65] reportaron dos casos en los que obtuvieron fecundación y desarrollo de embriones de buena calidad inyectando oocitos con espermátidas, lo cual puede significar una opción para aquellos pacientes con azoospermia no obstructiva que desean tener un hijo biológico.

Se conoce que diferentes condiciones que padecen algunos hombres pueden afectar negativamente la calidad de las muestras de semen; sin embargo, existen reportes de casos en donde, utilizando la inyección intracitoplasmática de espermatozoides, se han obtenido resultados aceptables en la fecundación de oocitos y las tasas de embarazo, y nacimientos de niños en pacientes receptores de trasplantes renales [66], hombres con lesiones medulares [67], un paciente con un trastorno genético conocido como deficiencia en la $5 \alpha$-reductasa 2 [68] y un paciente con un defecto de nacimiento llamado Síndrome de Eagle-Barret [69].

Además, en pacientes subfértiles, es decir, aquellos con al menos un parámetro seminal anormal, se demostró que la inyección intracitoplasmática de espermatozoides es una técnica más eficiente en términos de fecundación [70,7I], mientras que en los casos con teratozoospermia severa (menor o igual que el $4 \%$ de espermatozoides normales de acuerdo a la morfología estricta de Kruger) [72,73] esta técnica ha presentado tasas de fecundación más altas comparado con la fertilización in vitro convencional; además, embriones de buena calidad [70]. De igual manera, estos mismos resultados fueron encontrados en un estudio realizado con pacientes que tenían parámetros seminales en el límite [74]. Sin embargo, las anormalidades en la cabeza del espermatozoide y las vacuolas presentes pueden afectar los resultados en los ciclos de la inyección intracitoplasmática de espermatozoides [75,76]. 


\section{Conclusiones}

La inyección intracitoplasmática de espermatozoides es una técnica efectiva diseñada para tratar la infertilidad en pacientes con factor masculino severo, incluyendo pacientes sometidos a biopsias testiculares o punción del epidídimo, por lo tanto, todo el espectro de la infertilidad masculina se puede tratar. Así mismo, representa una opción para aquellas personas que desean un hijo biológico y están afectados por cualquiera de las condiciones patológicas ya mencionadas. Actualmente, representa el tratamiento de alta complejidad más utilizado en el mundo debido a su alta tasa de fecundación, su tasa de embarazo comparable a la fertilización in vitro convencional y la alta estandarización y eficacia demostrada. Uno de los factores determinantes en su éxito radica en el uso de instrumentos especializados como el microscopio invertido y el equipo de micromanipulación, que permiten el manejo de los gametos masculino y femenino, y en la experiencia y destreza del embriólogo encargado.

\section{Bibliografía}

I. Practice Committee of American Society for Reproductive Medicine. Definitions of infertility and recurrent pregnancy loss: a committee opinion. Fertil Steril 20 13; 99: 63.

2. Akhondali Z, Dianat M, Radan M. Negative Chronotropic and Antidysrhythmic Effects of Hydroalcoholic Extract of Lemon Balm (Melissa Officinalis L.) on $\mathrm{CaCl} 2$-Induced Arrhythmias in Rats. Electron Physician 2015; 7: 971-976.

3. Puzzielo L, Villasante A. Inducción de la ovulación y coito programado. En: Remohí J, Bellver J, Domingo J, Bosch E, Pellicer A, eds. Manual práctico de Esterilidad y Reproducción Humana: aspectos clínicos (ed 3ra). Madrid, España: Mc Graw Hill-Interamericana; 2008: |3-2|.

4. Sánchez I, Amorós D, Lucco F, González S, Ballesteros A, Pellicer A. Inseminación artificial. En: Remohí J, Bellver J, Domingo J, Bosch E, Pellicer A, eds. Manual práctico de Esterilidad y Reproducción Humana: aspectos clínicos (ed 3ra). Madrid, España: Mc Graw Hill-Interamericana; 2008: 23-40.

5. Cantineau AE, Janssen MJ, Cohlen BJ. Synchronised approach for intrauterine insemination in subfertile couples. Cochrane Database Syst Rev 2010 : CD006942.

6. Barros Delgadillo JC, Martínez Barrios E, Moreno Aburto C, Souraye M, Enríquez G, Manzur Navarrete $\mathrm{F}$, et al. Inseminación intrauterina versus coito programado en ciclos de hiperestimulación ovárica controlada. Ginecol Obstet Mex 2008; 76: 18-31.

7. Macklon NS, Broekmans FJ, Fauser BCJM. Indications for IVF treatment: from diagnosis to prognosis. En: Gardner DK, Weissman A, Howles CM, Shoham Z, eds. Textbook of Assisted Reproductive Technologies: Laboratory and Clinical Perspectives (ed 3ra). Reino Unido: Informa UK Ltd.; 2009: 447458.
8. Galan A, Campos P, Blanco C, Salinas R, Cobo A. Inseminación de los ovocitos. En: Remohí J, Cobo A, Romero L, de los Santos M, Pellicer A, eds. Manual práctico de Esterilidad y Reproducción Humana: laboratorio de reproduccion asistida (ed 3ra). Madrid, España: Mc Graw Hill-Interamericana; 2008: |5|-|56.

9. Palermo G, Joris H, Devroey P, Van Steirteghem AC. Pregnancies after intracytoplasmic injection of single spermatozoon into an oocyte. Lancet 1992; 340: 17-18.

10. Practice Committees of the American Society for Reproductive Medicine and Society for Assisted Reproductive Technology. Intracytoplasmic sperm injection (ICSI) for non-male factor infertility: a committee opinion. Fertil Steril 2012; 98: 13951399.

II. Palermo G, Neri Q, Takeuchi T, Hong S, Rosenwaks Z. Intracytoplasmic sperm injection: technical aspects. En: Gardner DK, Weissman A, Howles CM, Shoham Z, eds. Textbook of Assisted Reproductive Technologies: Laboratory and Clinical Perspectives (ed 3ra). Reino Unido: Informa UK Ltd.; 2009: I7I 180.

12. World Health Organization. WHO laboratory manual for the Examination and processing of human semen (ed 5a). Suiza: WHO Press; 2010.

13. Kupka MS, D'Hooghe T, Ferraretti AP, de Mouzon J, Erb K, Castilla JA, et al. Assisted reproductive technology in Europe, 201 I: results generated from European registers by ESHREdagger. Hum Reprod 2016; 31: 233-248.

14. Steptoe PC, Edwards RG. Birth after the reimplantation of a human embryo. Lancet 1978; 2: 366.

15. European Society of Human Reproduction and Embryology. TThe world's number of IVF and 
ICSI babies has now reached a calculated total of 5 million. 2012. Disponible: https://www.eshre. eu/Press-Room/Press-releases/Press-releases-ESHRE-20 I2/5-million-babies.aspx. Consultado: octubre 2015.

16. Ron-El R, Strassburger D, Friedler S, Komarovski $D$, Bern O, Soffer Y, et al. Extended sperm preparation: an alternative to testicular sperm extraction in non-obstructive azoospermia. Hum Reprod 1997; 12: 1222-1226.

17. Meniru GI. Intracytoplasmic sperm injection. En: Meniru Gl, ed. Cambridge guide to infertility management and assisted reproduction. Reino Unido: Cambridge University Press; 2004: |53- | 8 I.

18. Ishikawa T, Shiotani M, Izumi $Y$, Hashimoto $H$, Kokeguchi S, Goto S, et al. Fertilization and pregnancy using cryopreserved testicular sperm for intracytoplasmic sperm injection with azoospermia. Fertil Steril 2009; 92: 174- 179.

19. Tournaye $\mathrm{H}$. Management of male infertility by assisted reproductive technologies. Baillieres Best Pract Res Clin Endocrinol Metab 2000; 14: 423435.

20. Jones J, Horne G, Fitzgerald C. Who needs ICSI? A nationwide UK survey on ICSI use. Hum Fertil (Camb) 2012; 15: |44-149.

2I. Babayev SN, Park CW, Bukulmez O. Intracytoplasmic sperm injection indications: how rigorous? Semin Reprod Med 2014; 32: 283-290.

22. Fauser BC, Devroey P, Diedrich K, Balaban B, Bonduelle M, Delemarre-van de Waal HA, et al. Health outcomes of children born after IVF/ICSI: a review of current expert opinion and literature. Reprod Biomed Online 20।4; 28: I62- I 82.

23. Rubino P, Vigano P, Luddi A, Piomboni P. The ICSI procedure from past to future: a systematic review of the more controversial aspects. Hum Reprod Update 2016; 22: 194-227.

24. Rienzi LF, Ubaldi FM. Oocyte retrieval and selection. En: Gardner DK, Weissman A, Howles CM, Shoham Z, eds. Textbook of Assisted Reproductive Technologies: Laboratory and Clinical Perspectives (ed 3ra). Reino Unido: Informa UK Ltd.; 2009: 85102.

25. De Vos A. Intracytoplasmic sperm injection (ICSI). Hum Reprod 2000; 15 Suppl 4: 59-64.

26. Bourne H, Archer J, Edgar DH, Baker HWG. Sperm preparation techniques. En: Gardner DK, Weissman A, Howles CM, Shoham Z, eds. Textbook of Assisted Reproductive Technologies: Laboratory and Clinical Perspectives (ed 3ra). Reino Unido: Informa UK Ltd.; 2009: 53-66.

27. Sellés E, Pérez I, Pellicer J, López M. Capacitación espermática. En: Remohí J, Cobo A, Romero L, de los Santos M, Pellicer A, eds. Manual práctico de Esterilidad y Reproducción Humana: laboratorio de reproduccion asistida (ed 3ra). Madrid, España: Mc Graw Hill-Interamericana; 2008: 25-29.
28. Mortimer ST, Swan MA, Mortimer D. Effect of seminal plasma on capacitation and hyperactivation in human spermatozoa. Hum Reprod 1998; 13: 2139. 2146.

29. Zulategui J, Cobo A, Romero J, Galán A, Albert C, de los Santos M. Inyección intracitoplasmática de espermatozoides (ICSI). En: Remohí J, Cobo A, Romero L, de los Santos M, Pellicer A, eds. Manual práctico de Esterilidad y Reproducción Humana: laboratorio de reproduccion asistida (ed 3ra). Madrid, España: Mc Graw Hill-Interamericana; 2008: I65-176.

30. De Geyter C, De Geyter M, Behre HM. Assisted Reproduction. En: Nieschlag E, Behre HM, Nieschlag S, eds. Andrology: Male Reproductive Health and Dysfunction (ed 3ra). Berlín, Alemania: Springer-Verlag; 2010 : 470-497.

31. Palermo GD, Bedford JM. Micromanipulation of Human Gametes, Zygotes, and Embryos. En: Keel BA, May JV, De Jonge C], eds. Handbook of the Assisted Reproduction Laboratory: CRC Press; 2000: 22I-252.

32. Barratt CL. Semen analysis is the cornerstone of investigation for male infertility. Practitioner 2007; 25।: 8-10, 12, 15- 17.

33. Kumar N, Singh AK. Trends of male factor infertility, an important cause of infertility: A review of literature. J Hum Reprod Sci 2015; 8: 191-196.

34. Bonde JP, Ernst E, Jensen TK, Hjollund NH, Kolstad $\mathrm{H}$, Henriksen TB, et al. Relation between semen quality and fertility: a population-based study of 430 first-pregnancy planners. Lancet 1998; 352: | | $72-1177$

35. De los Ríos J, Cardona WD, Berdugo JA, Correa C, Arenas A, Olivera-Angel M, et al. Los valores espermáticos de 113 individuos con fertilidad reciente no mostraron correlación con los parámetros establecidos por la OMS. Arch Esp Urol 2004; 57: | 47- 152

36. Jequier AM. Clinical andrology--still a major problem in the treatment of infertility. Hum Reprod 2004; 19: 1245-1249.

37. van der Steeg JW, Steures P, Eijkemans MJ, JD FH, Hompes PG, Kremer JA, et al. Role of semen analysis in subfertile couples. Fertil Steril 201 I; 95 1013-1019.

38. Centola GM. Semen assessment. Urol Clin North Am 2014; 41: 163-167.

39. Cohen-Bacrie P, Belloc S, Menezo YJ, Clement P, Hamidi J, Benkhalifa M. Correlation between DNA damage and sperm parameters: a prospective study of I,633 patients. Fertil Steril 2009; 91: I80 I- | 805.

40. Goker EN, Sendag F, Levi R, Sendag H, Tavmergen E. Comparison of the ICSI outcome of ejaculated sperm with normal, abnormal parameters and testicular sperm. Eur J Obstet Gynecol Reprod Biol 2002; 104: 129-136. 
4I. Bukulmez O, Yucel A, Yarali H, Bildirici I, Gurgan T. The origin of spermatozoa does not affect intracytoplasmic sperm injection outcome. Eur J Obstet Gynecol Reprod Biol 200I; 94: 250-255.

42. Sukcharoen N, Sithipravej T, Promviengchai $S$, Chinpilas V, Boonkasemsanti W. Sperm morphology evaluated by computer (IVOS) cannot predict the fertilization rate in vitro after intracytoplasmic sperm injection. Fertil Steril 1998; 69: 564-568.

43. French DB, Sabanegh ES, Jr., Goldfarb J, Desai N. Does severe teratozoospermia affect blastocyst formation, live birth rate, and other clinical outcome parameters in ICSI cycles? Fertil Steril 2010; 93: 1097-1 103.

44. De Vos A, Van De Velde H, Joris H, Verheyen G, Devroey P, Van Steirteghem A. Influence of individual sperm morphology on fertilization, embryo morphology, and pregnancy outcome of intracytoplasmic sperm injection. Fertil Steril 2003; 79: 4248.

45. Keegan BR, Barton S, Sanchez X, Berkeley AS, Krey LC, Grifo J. Isolated teratozoospermia does not affect in vitro fertilization outcome and is not an indication for intracytoplasmic sperm injection. Fertil Steril 2007; 88: I583-|588.

46. Jiang Y, Cao Q-y, Li S-x, Meng F-y, Geng C-p, Song G. Impact of Semen Liquefaction and Viscosity on the Outcomes of in vitro Fertilization. J Reprod \& Cont 2012; 23: 169-178.

47. Bhattacharya S, Hamilton MP, Shaaban M, Khalaf $\mathrm{Y}$, Seddler M, Ghobara T, et al. Conventional in-vitro fertilisation versus intracytoplasmic sperm injection for the treatment of non-male-factor infertility: a randomised controlled trial. Lancet 200 I; 357: 2075-2079.

48. Briton-Jones CM, Buehler N, Danzer $H$, Surrey M, Hill DL. ICSI vs IVF in sibling oocytes on: fertilization rate, embryo cleavage rate, embryo quality and aneuploidy rates, from patients with primary unexplained infertility and normal semen analysis. Fertil Steril 2009; 92: S36.

49. De Brucker M, Camus M, Haentjens P, Francotte J, Verheyen G, Tournaye H. Cumulative delivery rates after ICSI with donor spermatozoa in different age groups. Reprod Biomed Online 2014; 28: 599 605.

50. Pregl Breznik B, Kovacic B, Vlaisavljevic V. Are sperm DNA fragmentation, hyperactivation, and hyaluronan-binding ability predictive for fertilization and embryo development in in vitro fertilization and intracytoplasmic sperm injection? Fertil Steril 2013; 99: 1233-1241.

5I. Wdowiak A, Bakalczuk S, Bakalczuk G. The effect of sperm DNA fragmentation on the dynamics of the embryonic development in intracytoplasmatic sperm injection. Reprod Biol 20 I5; 15: 94- 100.

52. loannou D, Miller D, Griffin DK, Tempest HG. Impact of sperm DNA chromatin in the clinic. J As- sist Reprod Genet 20 16; 33: 157- 166.

53. Wright C, Milne S, Leeson H. Sperm DNA damage caused by oxidative stress: modifiable clinical, lifestyle and nutritional factors in male infertility. Reprod Biomed Online 20।4; 28: 684-703.

54. Mayorga Torres JM, Peña B, Cadavid AP, Cardona Maya WD. La importancia clínica del ADN espermático en el análisis seminal cotidiano. Rev Chil Obstet Ginecol 20I5; 80: 265-268.

55. Agarwal A, Said TM. Role of sperm chromatin abnormalities and DNA damage in male infertility. Hum Reprod Update 2003; 9: 331-345.

56. Larson KL, DeJonge CJ, Barnes AM, Jost LK, Evenson DP. Sperm chromatin structure assay parameters as predictors of failed pregnancy following assisted reproductive techniques. Hum Reprod 2000; 15: 1717-1722.

57. Brahem S, Mehdi M, Landolsi H, Mougou S, Elghezal H, Saad A. Semen parameters and sperm DNA fragmentation as causes of recurrent pregnancy loss. Urology 201 I; 78: 792-796.

58. Gil-Villa AM, Cardona-Maya W, Agarwal A, Sharma R, Cadavid A. Assessment of sperm factors possibly involved in early recurrent pregnancy loss. Fertil Steril 2010; 94: |465-|472.

59. Oleszczuk K, Giwercman A, Bungum M. Sperm chromatin structure assay in prediction of in vitro fertilization outcome. Andrology 20 16; 4: 290-296.

60. Yeung C-H, Cooper TG. Sperm Quality and Function Tests. En: Nieschlag E, Behre HM, Nieschlag S, eds. Andrology: Male Reproductive Health and Dysfunction (ed 3ra). Berlín, Alemania: Springer-Verlag; 2010: |40-15|.

6I. Hammadeh ME, Radwan M, Al-Hasani S, Micu R, Rosenbaum P, Lorenz M, et al. Comparison of reactive oxygen species concentration in seminal plas$\mathrm{ma}$ and semen parameters in partners of pregnant and non-pregnant patients after IVF/ICSI. Reprod Biomed Online 2006; 13: 696-706.

62. Mayorga-Torres BJM, Cardona-Maya W, Cadavid Á, Camargo M. Evaluación de los parámetros funcionales espermáticos en individuos infértiles normozooespérmicos. Acta Urolog Español 2013; 37: $221-227$.

63. Gil-Salom M, Romero J, Rubio C, Ruiz A, Remohi J, Pellicer A. Intracytoplasmic sperm injection with cryopreserved testicular spermatozoa. Mol Cell Endocrinol 2000; 169: 15-19.

64. Gil Salom M. Técnicas de recuperación espermática para inyección intracitoplasmática de espermatozoides (ICSI) en infertilidad masculina. Arch Esp Urol 2004; 57: 1035-1046.

65. Goswami G, Singh S, Devi MG. Successful fertilization and embryo development after spermatid injection: A hope for nonobstructive azoospermic patients. J Hum Reprod Sci 20 I5; 8: 175- 177.

66. Berkkanoglu M, Bulut H, Coetzee K, Ozgur K. In- 
tracytoplasmic sperm injection in male renal transplant recipients. Middle East Fertil Soc J 2015; 20: 127-130.

67. Giulini S, Pesce F, Madgar I, Marsella T, Volpe A, de Aloysio D, et al. Influence of multiple transrectal electroejaculations on semen parameters and intracytoplasmic sperm injection outcome. Fertil Steril 2004; 82: 200-204.

68. Matsubara K, Iwamoto H, Yoshida A, Ogata T. Semen analysis and successful paternity by intracytoplasmic sperm injection in a man with steroid 5alpha-reductase-2 deficiency. Fertil Steril 20 10; 94 : 2770 e2777-2710.

69. Fleming SD, Varughese E, Hua VK, Robertson A, Dalzell F, Boothroyd CV. Normal live births after intracytoplasmic sperm injection in a man with the rare condition of Eagle-Barrett syndrome (prunebelly syndrome). Fertil Steril 20 13; I00: I532- 1535.

70. Pisarska MD, Casson PR, Cisneros PL, Lamb DJ, Lipshultz LI, Buster JE, et al. Fertilization after standard in vitro fertilization versus intracytoplasmic sperm injection in subfertile males using sibling oocytes. Fertil Steril 1999; 71: 627-632.

7I. Tournaye H, Verheyen G, Albano C, Camus M, Van Landuyt L, Devroey P, et al. Intracytoplasmic sperm injection versus in vitro fertilization: a randomized controlled trial and a meta-analysis of the literature. Fertil Steril 2002; 78: 1030-1037.

72. Kruger TF, Acosta AA, Simmons KF, Swanson RJ, Matta JF, Oehninger S. Predictive value of abnormal sperm morphology in in vitro fertilization. Fertil Steril 1988; 49: I 12-1 I7.

73. Van Waart J, Kruger TF, Lombard CJ, Ombelet $W$. Predictive value of normal sperm morphology in intrauterine insemination (IUI): a structured literature review. Hum Reprod Update 2001; 7: 495 500.

74. van der Westerlaken L, Naaktgeboren N, Verburg H, Dieben S, Helmerhorst FM. Conventional in vitro fertilization versus intracytoplasmic sperm injection in patients with borderline semen: a randomized study using sibling oocytes. Fertil Steril 2006; 85: 395-400.

75. Chaichian S, Tamannaie Z, Rohani H, Ahmadi M, Nasr MH, Pazouki A, et al. Relationship between sperm parameters and intracytoplasmic sperm injection outcome. Middle East Fertil Soc J 20I 5; 20: $25 \mathrm{I}-254$

76. Falagario D, Brucculeri AM, Depalo R, Trerotoli P, Cittadini E, Ruvolo G. Sperm head vacuolization affects clinical outcome in ICSI cycle. A proposal of a cut-off value. J Assist Reprod Genet 2012; 29: |28|-|287.

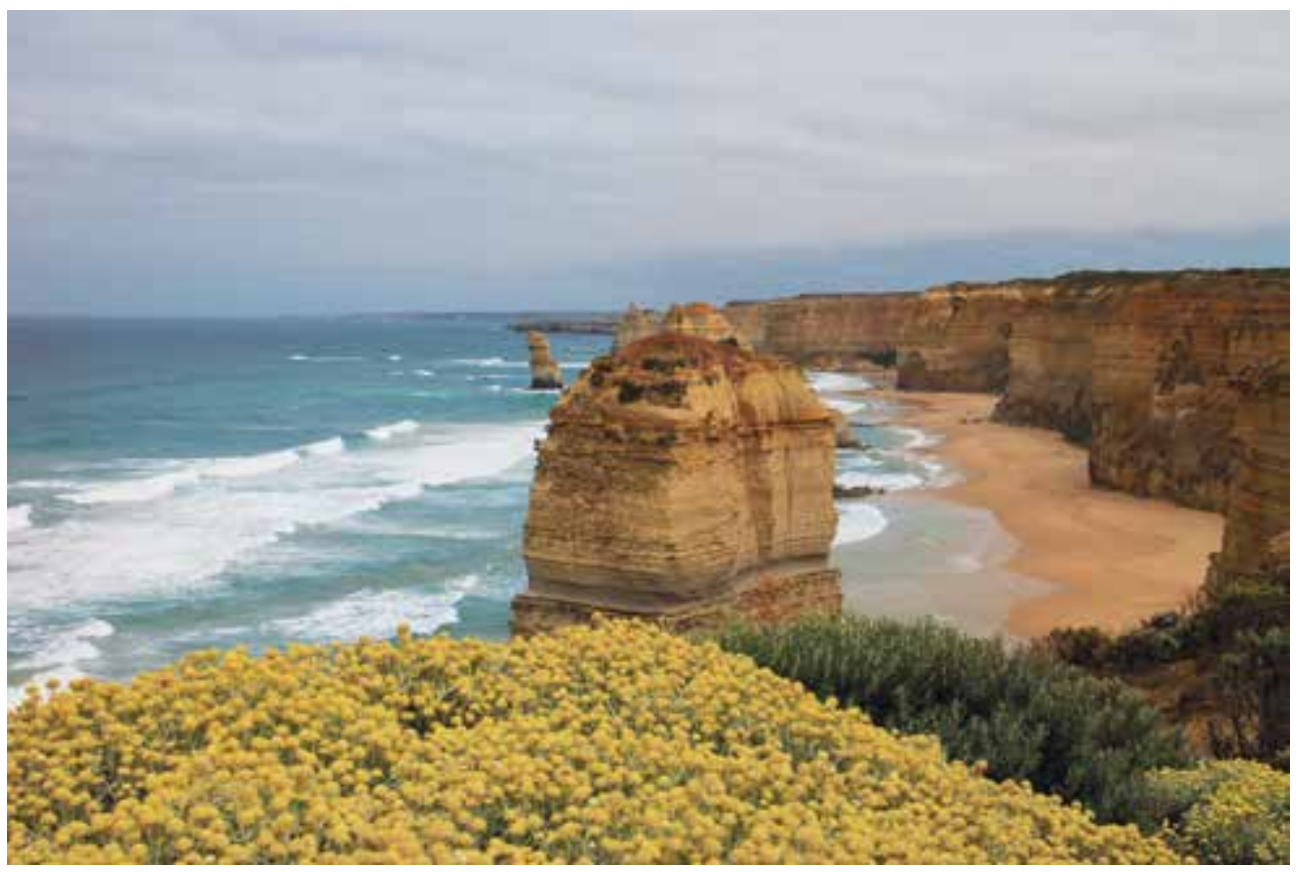

Great Ocean Road.

Australia.

Carlos Alberto Lozano M. carlos.lozano@iatm.com.co 